\title{
Ozonoterapia en medicina del dolor. Revisión
}

\author{
F. J. Hidalgo-Tallón ${ }^{1}$ y L. M. Torres ${ }^{2}$ \\ ${ }^{1}$ Instituto de Neurociencias. Universidad de Granada. ${ }^{2}$ Servicio de Anestesia, Reanimación y Tratamiento del \\ Dolor. Hospital Puerta del Mar. Cádiz
}

Hidalgo-Tallón FJ, Torres LM. Ozonoterapia en medicina del dolor. Revisión. Rev Soc Esp Dolor 2013; 20(6): 291300.

\begin{abstract}
Increasingly more medical ozone is used in the treatment of pain. So much so that recently the Spanish Ministry of Health has included ozone therapy in the portfolio of pain units.

The effectiveness, safety and good tolerability of ozone, infiltrated as well as administered systemically, justify the extent of its use in recent years.

Because little is known in general on this subject, the aim of this revision is to update current knowledge about ozone therapy in pain medicine.
\end{abstract}

Key words: Ozone. Medical ozone. Pain medicine.

\section{RESUMEN}

Cada vez se emplea más el ozono médico en el tratamiento del dolor. Tanto es así que recientemente el Ministerio de Sanidad español ha incluido la ozonoterapia en la cartera de servicios de las unidades de dolor.

La efectividad, seguridad y buena tolerabilidad del ozono, tanto infiltrado como administrado por vía sistémica, justifican la extensión de su uso en los últimos años.

Debido al escaso conocimiento que en general hay acerca del tema, pretendemos, con esta revisión, poner al día los conocimientos actuales en torno a la ozonoterapia en medicina del dolor.

Recibido: 05-02-13.

Aceptado: 07-04-13.
Palabras clave: Ozonoterapia. Ozono médico. Medicina del dolor.

\section{INTRODUCCIÓN}

La ozonoterapia en medicina es una realidad, y cada vez hay más profesionales haciendo uso del ozono médico como complemento terapéutico para diferentes enfermedades relacionadas con el estrés oxidativo, incluido el dolor crónico.

Ya existe una organización médica en torno a la ozonoterapia, y hay asociaciones profesionales que garantizan la buena praxis, y se enseña la ozonoterapia en másteres y cursos de experto de diversas universidades (Curso de experto universitario en ozonoterapia, Universidad de Sevilla, www.aepromo.org, www.seot.es, www.aceoot. org).

Se calcula que en el mundo hay más de 26.000 médicos expertos en ozonoterapia, y en Europa son tratados anualmente en torno a los 10 millones de pacientes (1).

Recientemente, el Ministerio de Sanidad español ha incluido la ozonoterapia en la cartera de servicios de las unidades de dolor, por lo que se hace necesario que el médico experto en el tratamiento del dolor conozca el fundamento científico de la ozonoterapia médica y cómo actúa, tanto a nivel local como sistémico.

El ozono es una molécula formada por tres átomos de oxígeno $\left(\mathrm{O}_{3}\right)$ en lugar de los dos de los que se compone la molécula de oxígeno $\left(\mathrm{O}_{2}\right)$. La ozonoterapia consiste en la aplicación de una mezcla de oxígeno médico con ozono; la mezcla ha de ser producida in situ para cada aplicación, y en ella nunca habrá más de un $5 \%$ de ozono. 
Las aplicaciones de ozono médico se remontan a principios del siglo pasado. El Dr. Kellogg, en su libro sobre difteria (1881) ya mencionaba el ozono como desinfectante, y en 1898 los doctores Thauerkauf y Luth fundaron en Berlín el Instituto para oxigenoterapia, llevando a cabo los primeros ensayos con animales. En el año 1911, salió a la luz el libro A Working Manual of High Frequency Currents, publicado por el Dr. Noble Eberhart, jefe del Departamento de Terapéutica Fisiológica de la Universidad de Loyola, donde se hablaba del uso del ozono médico en el tratamiento de enfermedades como la tuberculosis, la anemia, el asma, la bronquitis, la fiebre del heno, la diabetes, etc. (2).

Pero a pesar de los éxitos obtenidos a principios del siglo pasado, las máquinas generadoras de ozono carecían de precisión, y es la tecnología actual la que nos permite obtener con toda fiabilidad la mezcla idónea de ambos gases.

En líneas generales, al aplicar este tipo de terapia, realmente estamos induciendo una "microoxidación" controlada e inocua, cuya respuesta orgánica será una activación favorable del sistema antioxidante celular.

Numerosos autores han trabajado a nivel preclínico sobre los efectos de la ozonoterapia sobre los organismos vivos, pudiéndose demostrar efectos beneficiosos sobre la capacidad antioxidante celular y la adaptación ante los procesos de isquemia/reperfusión (3-7).

Desde un punto de vista clínico, la ozonoterapia presenta múltiples aplicaciones médico-quirúrgicas, todas ellas relacionadas con la capacidad germicida del ozono, con los procesos isquémicos y con las descompensaciones del balance redox celular. Son varios los manuales médicos que recogen la experiencia y los trabajos científicos llevados a cabo hasta la fecha por diferentes grupos de investigación, principalmente italianos, alemanes, rusos y cubanos $(1,8,9)$.

Las formas de aplicación del ozono médico son básicamente tres: tópica, infiltrativa y sistémica.

Las aplicaciones tópicas sacan partido del poder germicida del ozono y de su efecto positivo sobre los procesos de cicatrización; se suele aplicar directamente, con el uso de bolsas de cierre hermético, o mediante agua o aceites ozonizados.

El ozono infiltrado a concentraciones de entre $4 \mathrm{y}$ $30 \mu \mathrm{g} / \mathrm{ml}$ es útil para tratar afecciones del aparato locomotor, tales como artritis, tendinitis, miositis, fascitis o dolores miofasciales.

La ozonoterapia sistémica consiste en la administración de la mezcla de gases fundamentalmente mediante dos vías: la autohemoterapia y la insuflación rectal. La autohemoterapia puede ser "mayor" o "menor". La autohemoterapia mayor consiste en la extracción de una cantidad determinada de sangre, que sin salir de un circuito cerrado es puesta en contacto con el gas, con el que reaccionará hasta la dilución del mismo; tras unos minutos la sangre se reinfunde. En la "autohemoterapia menor" la mezcla tiene lugar en una jeringa, y la sangre ozonizada se inyecta por vía intramuscular.

Mediante la insuflación rectal, la mezcla de gases es amplia y rápidamente absorbida a través de la mucosa intestinal; esta técnica resulta fácil de aplicar, barata, muy segura y cómoda.

\section{INFILTRACIONES CON OXÍGENO/OZONO MÉDI- $\mathrm{CO}\left(\mathrm{O}_{2} / \mathrm{O}_{3}\right)$}

\section{Generalidades}

El uso de las infiltraciones de $\mathrm{O}_{2} / \mathrm{O}_{3}$ para tratar patología músculo-articular cada vez está más extendido.

C. Verga (10) fue el primero en describir las aplicaciones de ozono intramuscular, a nivel paravertebral y en los puntos gatillo, en pacientes con lumbalgia crónica. Posteriormente, en la década de los 90, se extiende su uso para tratar poliartritis aguda y crónica (cadera, rodilla, articulación sacroiliaca, interfalángicas), tendinitis, epicondilitis, síndrome del túnel carpiano y dolores miofasciales (9).

A pesar de su uso cada vez más frecuente, los niveles de evidencia (a excepción del tratamiento de la hernia discal) son escasos, posiblemente por limitación casi exclusiva de su uso en el ámbito de la medicina privada. Carmona (11) realizó una revisión sistemática en el año 2006 sobre la efectividad de la ozonoterapia en las enfermedades reumáticas en general, concluyendo que no existen ensayos clínicos de calidad, que la mayoría de los trabajos están publicados en revistas de bajo impacto y que la metodología entre los distintos estudios es muy variable.

Pero aunque los ensayos clínicos sean escasos, sí hay trabajos científicos menores ilustrando el empleo del ozono infiltrado como analgésico-antiinflamatorio en diversas patologías del aparato locomotor $(12,13)$.

\section{Ozonoterapia en patología de rodilla}

Riva Sanseverino registró 156 pacientes con patología articular de rodilla (artritis postraumática, gonartrosis con deformidad leve y gonartrosis con deformidad severa) obteniendo buenos resultados, que fueron especialmente beneficiosos cuando no había deformidades óseas severas. El tratamiento consistió en infiltraciones intraarticulares y periarticulares de $10 \mathrm{ml}$ de oxígeno/ozono a una concentración de $20 \mu \mathrm{g} / \mathrm{ml}$ (14).

En Cuba, en el año 1997, se llevó a cabo un estudio prospectivo para evaluar la efectividad de las infiltraciones de ozono en 126 pacientes con osteoartritis de rodilla; por lo general, se necesitaron 3 o 4 infiltraciones para obtener resultados positivos, y solo a 14 pacientes se le aplicaron más de 5 sesiones. El 71,4\% de los pacientes tuvieron un 
resultado catalogado como bueno, en el 10,3\% el resultado fue regular, y en un 18,3\% el resultado fue malo. La principal complicación fue el dolor durante la infiltración, y es de destacar el ahorro económico por la menor necesidad de antiinflamatorios (15).

La condromalacia femoropatelar es una patología dolorosa cuyo tratamiento es fundamentalmente quirúrgico, tras el que con frecuencia quedan secuelas. Manzi y Raimondi trataron con $\mathrm{O}_{2} / \mathrm{O}_{3}$ a 60 pacientes refractarios al tratamiento quirúrgico convencional, obteniendo una resolución del cuadro mayor y más rápida que en los controles (16).

También se ha usado con éxito la ozonoterapia infiltrada a nivel peritendinoso en tendinopatías refractarias de rodilla (17). Gjonovich y su equipo mejoraron a 36 atletas con "rodilla de saltador" que no habían respondido a los tratamientos convencionales (18).

Moretti y colaboradores, en la osteoartritis temprana de rodilla, compararon la efectividad de la infiltración de $\mathrm{O}_{2} /$ $\mathrm{O}_{3}$ con la de ácido hialurónico, concluyendo que si bien no había diferencias estadísticamente significativas, estaría más indicado el ozono en estadios tempranos, donde predomina la inflamación (19).

\section{Ozonoterapia en patología de hombro}

Con respecto a la patología de hombro, Ikonomidis y colaboradores demostraron en un ensayo clínico la mayor efectividad del $\mathrm{O}_{2} / \mathrm{O}_{3}$ frente a las infiltraciones con esteroides o la terapia con ultrasonidos en una serie de infiltraciones subacromiales y en las zonas de máximo dolor (20). También se ha empleado con éxito la oxígeno-ozonoterapia, en combinación con las ondas de choque, para tratar tendinitis calcificada del hombro (21), y Brina y Villani han publicado la utilidad de las infiltraciones ecoguiadas de $\mathrm{O}_{2} /$ $\mathrm{O}_{3}$ en pacientes con lesiones del manguito de los rotadores no quirúrgicas (22).

\section{Ozonoterapia en patología de columna}

Sin duda, la mayor cantidad de trabajos publicados se centra en el uso de la ozonoterapia para el tratamiento de la hernia discal, tanto a nivel cervical como lumbar.

Generalmente, el tratamiento de las hernias cervicales es más conservador que el de las lumbares, y su mayor complejidad hace que muchos menos pacientes sean derivados a cirugía. En este contexto el interés de las infiltraciones intradiscales o paravertebrales de oxígeno/ozonoterapia tiene una relevancia especial, y los efectos analgésicos, antiinflamatorios y relajantes musculares de la ozonoterapia en la patología cervical han sido descritos $(23,24)$.

En 2004, Moretti y su grupo realizaron un ensayo clínico comparando la efectividad de la ozonoterapia con la de la mesoterapia en pacientes con dolor cervical, parestesias de miembros superiores (uni o bilaterales), vértigo periférico y cefalea. Se reclutaron 152 pacientes con hernias, protrusiones o cervicoartrosis, 76 de los cuales fueron tratados con infiltraciones de $\mathrm{O}_{2} / \mathrm{O}_{3}$ a nivel muscular paravertebral, en los trapecios y en los elevadores de la escápula; a los otros 76 pacientes se les aplicó tratamiento con mesoterapia antiinflamatoria. Las diferencias fueron estadísticamente significativas a favor del grupo tratado con oxígeno-ozono, con un $78 \%$ de resultados calificados como buenos u óptimos, frente a un 56,25\% en el grupo de la mesoterapia (25).

En cuanto a las infiltraciones intradiscales a nivel cervical, también demostraron ser eficaces, según un trabajo publicado por Yue Yong Xiao y colaboradores. Se hizo una evaluación retrospectiva de 86 pacientes con espondilosis tratados con infiltraciones guiadas por tomografía, de los que 37 sufrían de mielopatía, 30 presentaban radiculopatía y 19 tenían sintomatología de tipo simpático. Las indicaciones para el tratamiento fueron el presentar cervicalgia con patrones de irradiación braquial, el tener pérdida de sensibilidad, hormigueo, entumecimiento, debilidad muscular o deficiencia de los reflejos tendinosos profundos; también se incluyeron pacientes con electromiografía $u$ otras pruebas de conducción eléctrica positivas, y todos habrían de ser refractarios a tratamiento con las terapias conservadoras al menos durante 12 semanas. Se descartaron los pacientes con estenosis ósea de canal, osificación del ligamento longitudinal posterior o malacia espinal. El tratamiento con ozonoterapia resultó ser excelente, bueno o pobre en el $78 \%, 16 \%$ y $6 \%$ de los casos, respectivamente, según se registró con el método $\mathrm{McNab}$ modificado (26).

En patología lumbar la cantidad de trabajos es extensa. Los resultados positivos, junto con la seguridad de la técnica y las altas tasas de fracaso posquirúrgico, hacen que cada vez más autores consideren de primera elección los tratamientos conservadores con ozonoterapia, sea paravertebral o intradiscal.

Muto y su equipo realizaron infiltraciones guiadas con TAC a 2.900 pacientes con hernia discal. El gas se inyectó a nivel intradiscal, periganglionar y perirradicular. Al mes se revisaron los pacientes, repitiendo la sesión en aquellos casos en que la mejoría fue parcial. A los 6 y 12 meses hubo mejorías del 75-80\% de los pacientes con hernia discal simple, del $70 \%$ con hernias múltiples y del $55 \%$ con dolor por cirugía fallida de espalda (27).

Cosma y sus colaboradores añadieron $\mathrm{O}_{2} / \mathrm{O}_{3}$ intradiscal y periganglionar a la infiltración con anestésicos locales y corticoides; no solo no hubo interferencia del gas sobre la efectividad de los medicamentos, sino que en la evaluación a los seis meses por evaluadores ciegos se pudo constatar una mejoría estadísticamente significativa con la combinación de ambos tratamientos (28).

Bonetti y su equipo, en un ensayo clínico, compararon la efectividad de la infiltración intraforaminal de $\mathrm{O}_{2} / \mathrm{O}_{3}$ con la 
infiltración perirradicular de esteroides. Se reclutaron 306 pacientes, con y sin enfermedad discal, que fueron divididos en dos grupos (166 y 140, respectivamente). El principal instrumento de medida fue el índice de McNab modificado, y se registró la evolución a corto (1 semana), medio (3 meses) y largo plazo (6 meses). A corto plazo no hubo diferencias estadísticamente significativas entre las dos modalidades de tratamiento $(p=0,4077)$. A largo plazo, las diferencias a favor de los tratamientos con ozono sí fueron estadísticamente significativas, pero solamente en el grupo de pacientes con enfermedad discal $(\mathrm{p}=0,0021)$; también a largo plazo se pudo ver que los tratamientos con ozonoterapia tenían estadísticamente menos índice de fracaso $(8,6 \%)$ que los tratamientos con esteroides (21,4\%) (29).

Autores como Torres y su equipo obtienen resultados positivos y duraderos en el tratamiento de la ciática por hernia discal aplicando tres sesiones consecutivas de infiltraciones de $\mathrm{O}_{2} / \mathrm{O}_{3}$ intradiscal, epidural y paravertebral que añaden a bupivacaína ( $5 \mathrm{ml}$ al $0,25 \%$ ) y triamcinolona $(4 \mathrm{mg})$. Aunque el estudio es retrospectivo, se registró la evolución de 91 pacientes durante 24 meses, con una mejoría muy significativa que persistió al final del seguimiento en el $81,1 \%$ de la muestra (30).

Buric y su grupo hicieron un seguimiento prospectivo durante 18 meses de 104 pacientes con protrusiones discales, encontrando mejorías en el dolor y la capacidad funcional en la mayoría de la muestra; se hicieron mediciones del volumen discal y se observó que a los 5 meses un $22 \%$ de las protrusiones no habían cambiado de volumen, un $41 \%$ se habían reducido y un $37 \%$ habían desaparecido (31). Los resultados indicaban que la técnica era efectiva en el tratamiento de las protrusiones, aunque según otro estudio posterior del mismo equipo, la efectividad no fue superior a la de la microdiscectomía (32).

También respecto a las protrusiones discales, He Qing y colaboradores, con una muestra de 602 pacientes y 1.078 discos intervenidos, concluyeron la idoneidad del tratamiento con ozonoterapia como de primera elección después de fallar técnicas más conservadoras. Al comparar la ozonoterapia con otras técnicas microinvasivas, estos autores consideraron que se trataba de una técnica efectiva, segura, mínimamente estresante para el paciente y fácil de realizar (33). Resultados igualmente positivos obtienen Castro y su equipo, en un estudio observacional prospectivo en el que trataron a 41 pacientes con infiltraciones simultáneas intradiscales, epidurales y perirradiculares. Se excluyeron enfermos con hernia discal con fragmento libre y déficit neurológico mayor asociado. La evolución fue muy positiva (según la EVA y el test de Lattinen) desde el primero al último de los registros posbasales (a los 30 días y a los 6 meses, respectivamente), y el grado de satisfacción fue calificado como bueno por el $85,4 \%$ de la muestra (34).

En el año 2006 se publicó otro ensayo clínico aleatorizado comparando la efectividad de la infiltración paraver- tebral muscular de $\mathrm{O}_{2} / \mathrm{O}_{3}$ con la de esteroides epidurales en pacientes refractarios a los tratamientos convencionales (esteroides y miorrelajantes). Fueron tratados con esteroides epidurales 171 pacientes y a 180 se le realizaron infiltraciones paravertebrales de oxígeno/ozono. A las tres semanas de seguimiento, la mejoría fue estadísticamente significativa a favor de los pacientes tratados con ozonoterapia (remisión total o casi total del dolor en el 88,2\%, frente al $59 \%$ en el grupo de los esteroides), y a los seis meses la evolución fue excelente o buena en el 77,1 \% de los pacientes tratados con ozonoterapia, frente al $47,3 \%$ de pacientes tratados con esteroides (35).

Recientemente se realizó un ensayo clínico para evaluar la efectividad de las infiltraciones paravertebrales de oxígeno/ozono en el tratamiento del dolor lumbar agudo con hernia discal. Aunque la mayoría de los trabajos publicados se refieran a las técnicas intradiscales, la realidad es que esta técnica, por su seguridad y simplicidad suele ser la más empleada en la práctica clínica. Se reclutaron 60 pacientes que fueron aleatorizados en dos grupos; a uno se trató con infiltraciones reales y en el otro estas fueron simuladas. Se hizo un seguimiento a los $15,30,90$ y 180 días tras el registro basal. Se observó que en los pacientes tratados mejoraron significativamente el dolor y la limitación funcional $(\mathrm{p}<0,05)$, necesitando menos medicación analgésica (36).

Finalmente, en el año 2010 se publicó un metaanálisis sobre la efectividad y seguridad de la ozonoterapia para el tratamiento de las hernias discales de cualquier tipo. Se incluyeron 12 estudios con una muestra total de 8.000 pacientes; las mejorías medias registradas fueron similares a las descritas para discectomía: 3,9 puntos sobre 10 en la escala visual analógica de dolor, 25,7 puntos en la capacidad funcional según el Índice de Discapacidad de Oswestry (ODI) y un 79,7 \% de mejoría en los registros de la escala $\mathrm{McNab}$ modificada. El porcentaje de complicaciones fue del $0,064 \%$, por lo que el tratamiento se consideró seguro y eficaz (37).

Alrededor de un $80 \%$ de la población en países occidentales experimentará al menos un episodio de lumbalgia a lo largo de su vida, y en el $55 \%$ de estos habrá un dolor radicular asociado (38). El síndrome de cirugía fallida de espalda oscila entre un 15 y un $20 \%$, lo que lleva a proponer tratamientos más conservadores y menos invasivos, como la ozonoterapia, cuya efectividad parece oscilar entre un 65 y un $80 \%$ sugiriendo que un mínimo cambio en el volumen discal se puede traducir en un gran cambio clínico (39). También se deberán tener en cuenta las fibrosis epidurales, los desgarros perineurales, las adherencias nerviosas, las limitaciones de la biomecánica por fibrosis y espasmos de los músculos paravertebrales y los síndromes miofasciales asociados (40).

En este contexto, las infiltraciones con $\mathrm{O}_{2} / \mathrm{O}_{3}$, tanto a nivel paravertebral profundo y de los puntos gatillo de la 
musculatura relacionada, como la nucleolisis percutánea con ozono, son técnicas en auge debido a su inocuidad, efectividad, facilidad de ejecución y bajo coste. Andreula y colaboradores (41), al comparar la nucleolisis con ozono con la nucleolisis enzimática, concluyen que, siendo los resultados clínicos similares, el tratamiento con ozonoterapia sería de primera elección debido a ventajas como las que siguen (Tabla I).

TABLA I. VENTAJAS DE LA OZONOTERAPIA EN LA NUCLEOLISIS

- No existe la posibilidad de que se den reacciones alérgicas o anafilácticas

- Posibilidad de repetir el tratamiento las veces que se considere

- Menor riesgo de infecciones, debido a las propiedades germicidas del ozono

- Posibilidad de usar una aguja más fina y, por lo tanto, menos traumática

- Menos molestias postinfiltración (2, 3 días frente a 1 o 2 semanas)

A estas ventajas se añadirían las descritas sobre los corticoides. A este respecto, Schwartz y colaboradores (1), en su guía para el uso médico del ozono, llaman la atención sobre los siguientes puntos (Tabla II).

TABLA II. VENTAJAS DEL OZONO INFILTRADO FRENTE A LOS CORTICOIDES

- El oxígeno-ozono médico no debilita tendones ni ligamentos

- No existe la posibilidad de que se dé artropatía por cristales

- La ozonoterapia no facilita la destrucción articular

- No se favorece la necrosis avascular (más bien sería una indicación)

- El ozono se puede infiltrar en campos infectados (ayudando a resolver el cuadro)

- La ozonoterapia no interfiere con otras patologías médicas: trastorno de ansiedad, osteoporosis, hipertensión, diabetes, obesidad, gastropatías, insuficiencia renal o insuficiencia hepática

- No hay un límite en el número de aplicaciones

- El ozono es más económico

Por último, hay que mencionar que queda por demostrar la efectividad de la ozonoterapia en el tratamiento del síndrome de cirugía fallida de espalda, altamente pre- valente entre los pacientes operados de columna, y que suele empeorar con las reentradas quirúrgicas. En estos enfermos se organizan fibrosis por cicatrices epidurales y perineurales, contracturas y espasmos paraespinales y diversas adherencias, cuyo estímulo inflamatorio crónico daría lugar a fenómenos neuroplásticos con sensibilización central y periférica. Teóricamente, las propiedades fibrinolíticas, antiinflamatorias y antioxidantes del $\mathrm{O}_{2} / \mathrm{O}_{3}$ infiltrado lo harían idóneo para el tratamiento de estos procesos. El equipo del Centro Médico Nacional 20 de Noviembre, de México DF, ha publicado recientemente dos trabajos tratando en cada caso a 30 pacientes. En ambos estudios se aplicó una primera sesión de epidural más paravertebrales seguida de tres sesiones semanales de paravertebrales; se emplearon dosis de $20 \mathrm{ml}$, a $30 \mu \mathrm{g} / \mathrm{ml}$ en la primera serie y a $50 \mu \mathrm{g} / \mathrm{ml}$ en la segunda, pero los tratamientos no pudieron mejorar el dolor de los pacientes $(42,43)$. De todas maneras, dada la envergadura del problema, más estudios, quizás con unos protocolos más extensos, se hacen necesarios a este respecto.

\section{Ozonoterapia infiltrativa en artritis reumatoide}

Un estudio preclínico llevado a cabo en el hospital de Nanfang comparó los efectos de las infiltraciones de oxígeno médico y de diferentes concentraciones de ozono; los autores demostraron que el ozono intraarticular infiltrado a una concentración de $40 \mu \mathrm{g} / \mathrm{ml}$ es capaz de inhibir la sinovitis en ratas con artritis reumatoide (44). Los médicos expertos en ozonoterapia emplean empíricamente las infiltraciones articulares desde hace años, supuestamente con resultados muy positivos, pero aún no existen trabajos de envergadura al respecto.

\section{Otras aplicaciones del oxígeno/ozono infiltrado}

Otras aplicaciones descritas son las diversas tendinopatías y síndromes de atrapamiento $(45,46)$, el tratamiento de epicondilitis (47), las espondilolistesis y espondilólisis (48), la espondilodiscitis con infección del espacio discal (49), la espondiloartrosis (50), la patología dolorosa del pie de atleta (51), la tenosinovitis de De Quervain (52), la metatarsalgia por fibrosis posquirúrgica tras resección de un neuroma de Morton (53) o la patología de la articulación temporomandibular (54).

\section{Propiedades terapéuticas y mecanismos de acción del ozono médico infiltrado}

Cuando infiltramos la mezcla de oxígeno/ozono, estamos infiltrando un gas altamente oxidante, con una buena 
capacidad de difusión tisular, que va a reaccionar con los fluidos intersticiales produciendo peróxido de hidrógeno $\left(\mathrm{H}_{2} \mathrm{O}_{2}\right)$ y radical hidroxilo $(\mathrm{OH} \cdot)$, además de otras especies reactivas del oxígeno (ERO), sean o no de naturaleza radical. Como respuesta, en el medio habrá un aumento de los sistemas antioxidantes, tales como la superóxido dismutasa (SOD), la glutatión peroxidada (GSH-Px) y la catalasa (CAT).

Diversos autores han descrito el efecto antiinflamatorio, analgésico, antiedema y descongestionante de las infiltraciones de $\mathrm{O}_{2} / \mathrm{O}_{3}$, y se estipula que la oxidación de los receptores algogénicos (14) inhibiría la señal dolorosa y activaría el sistema antinociceptivo. Estas propiedades favorecerían un efecto relajante muscular, así como la mejoría de la movilidad de la zona tratada que se puede observar en clínica (55). Todo esto es muy importante en la recuperación muscular con infiltraciones de $\mathrm{O}_{2} / \mathrm{O}_{3}$; Balkanyi (56) ha descrito la utilidad de la ozonoterapia en el tratamiento de la hipertonía muscular dolorosa, destacando el tremendo efecto relajante muscular que se produce.

Periféricamente el ozono sería capaz de inactivar los enzimas proteolíticos. Al mismo tiempo, mediada por el $\mathrm{H}_{2} \mathrm{O}_{2}$, se estimularía la proliferación de fibroblastos y condrocitos, favoreciendo la regeneración cartilaginosa.

Se sugiere que los efectos del ozono sobre la sopa bioquímica inhibirían la acción de las prostaglandinas proinflamatorias, la serotonina, la bradiquinina o la sustancia $\mathrm{P}$, justificando así su efecto analgésico, antiinflamatorio y remielinizante.

La acción antiinflamatoria del $\mathrm{O}_{2} / \mathrm{O}_{3}$ también se potenciaría por la inhibición de las citoquinas proinflamatorias (IL1, IL6, IL8, IL12, IL15 y TNF $\alpha$ ) y de la fosfolipasa A2, y por la acción estimulante de las citoquinas inmunosupresoras, tales como la IL10 y el TNF $\beta 1$ (de efecto antiinflamatorio y reparador tisular) (9).

El ozono infiltrado a nivel subcutáneo interaccionaría con los mediadores y/o los receptores periféricos del dolor, que serían oxidados (57). La infiltración subcutánea de $\mathrm{O}_{2} /$ $\mathrm{O}_{3}$ produciría cambios periféricos que inducirían neuroplasticidad central, según se ha podido observar en estudios preclínicos en los que se ha medido la regulación de la expresión de genes que juegan un papel esencial en el desarrollo y el mantenimiento de la alodinia. A este respecto, Fuccio y su equipo, al inducir un daño ciático en ratones comprobaron la activación corticofrontal de genes caspasa 1,8 y 12 (proinflamatorios, proapoptóticos y responsables de la alodinia); esta expresión se normalizó con una única inyección periférica de $\mathrm{O}_{2} / \mathrm{O}_{3}$, con lo que también se redujo la alodinia mecánica (58).

En cuanto al núcleo pulposo del disco intervertebral, se ha descrito que el $\mathrm{H}_{2} \mathrm{O}_{2}$, el HO y las ERO reaccionarán con los aminoácidos e hidratos de carbono de los proteoglicanos y colágeno I y II que conforman la matriz, dando lugar a un proceso de "momificación", que encogería el disco disminuyendo la compresión (59-63). Iliakis y su grupo estudiaron los cambios histológicos de la matriz tras un tratamiento con discólisis mediante ozono a una concentración de $27 \mu \mathrm{g} / \mathrm{ml}$. A nivel preclínico ensayaron los efectos de la discólisis con conejos, y a nivel clínico examinaron especímenes discales resecados mediante microdiscectomía. A las cinco semanas no se observaban signos de hiperplasia condrocítica perilesional ni infiltrados inflamatorios propios de las biopsias en los discos herniados; sí había un tejido fibroso de menos volumen ("disco momificado"), lo que supone una disminución de la compresión sobre la raíz nerviosa, una disminución del éxtasis venoso, una mejoría de la circulación, una mayor oxigenación y menos dolor, dada la gran sensibilidad a la hipoxia de las raíces nerviosas (64).

También hay que considerar la importancia del TNF $\beta 1$ en la síntesis del colágeno y los glicosaminoglicanos $(65,66)$, efecto que ha sido comprobado en la reparación tras la discectomía, con la reorganización del núcleo pulposo residual a expensas de una fibrosis incipiente.

\section{Dosificación}

En cuanto a la dosificación, se echan en falta protocolos estandarizados. La mayoría de los autores acoplan la cantidad de la mezcla gaseosa a la extensión del terreno a infiltrar o a la cavidad articular de la que se trate. Generalmente, las cantidades de gas oscilan entre los 5 y los $15 \mathrm{ml}$, a unas concentraciones de ozono que varían entre los 4 y los $30 \mu \mathrm{g} / \mathrm{ml}$.

El número de sesiones de infiltración suele ser de unas 10 o 12 (por lo general, dos por semana) cuando se trata de procesos inflamatorios crónicos del aparato locomotor, aunque en general las sesiones se suelen ajustar a la evolución concreta de cada caso.

Con respecto a las discólisis, se suele asumir que un caso no responde al tratamiento cuando se han realizado un máximo de dos o tres intervenciones. Torres y colaboradores, tras haber empleado diferentes concentraciones con el mismo protocolo clínico, observan mejorías más evidentes cuando infiltran el $\mathrm{O}_{2} / \mathrm{O}_{3}$ intradiscal a $50 \mu \mathrm{g} / \mathrm{ml}$ en comparación a cuando usan concentraciones inferiores, a 30 y $40 \mu \mathrm{g} / \mathrm{ml}(30)$.

\section{OZONOTERAPIA SISTÉMICA EN MEDICINA DEL DOLOR}

Como se ha comentado, la ozonoterapia estaría indicada, como coadyuvante, en el tratamiento de las enfermedades que cursan con alteraciones del balance redox celular o de la oxigenación tisular. Desde este punto de vista, la ozonoterapia sistémica ayudaría al paciente con dolor crónico, ya 
que estudios preclínicos recientes han demostrado el papel de las especies reactivas del oxígeno (ERO) en la hiperalgesia, vía activación de los receptores N-metil-D-aspartato (NMDA). Gao y su equipo (67), en un modelo preclínico de dolor, tanto neuropático como inflamatorio, pudieron demostrar que las ERO a nivel del asta dorsal acompañaban al dolor, y que la administración sistémica de un agente neutralizador de las mismas reducía la hiperalgesia mediante el bloqueo de la fosforilación desde los NMDA. Posteriormente, el mismo grupo de investigación (induciendo hiperalgesia por capsaicina en ratas) pudo demostrar el papel del anión superóxido como responsable del procesamiento anormal de la señal dolorosa en el asta dorsal, sugiriendo el papel terapéutico de la superóxido dismutasa mitocondrial (SOD-2) en estos tipos de dolor (68).

Pero, ciertamente, los niveles de evidencia científica en el tratamiento con ozonoterapia sistémica del dolor crónico son prácticamente inexistentes. A este respecto, solo se pueden recopilar algunas referencias de trabajos presentados a congresos.

Teóricamente, el efecto beneficioso sobre pacientes inmunodeprimidos y la capacidad germicida del ozono lo harían útil, tanto en el tratamiento de la infección por herpes zóster como de la neuralgia posherpética. En este campo son varios los expertos que han ensayado tratamientos con ozono durante años, al parecer con resultados positivos. Generalmente se usa la ozonoterapia como coadyuvante a los tratamientos convencionales, bien vía sistémica o local (infiltraciones o aplicaciones de aceites y agua ozonizada), pero podemos pensar que los defectos de protocolización y comunicación relegan estos trabajos al nivel de una anécdota médica, de utilidad para incentivar el desarrollo de trabajos mejor diseñados (69-71).

El ozono sistémico también se ha ensayado, de manera complementaria a las infiltraciones, en pacientes con artritis reumatoide. Menéndez y colaboradores añadieron satisfactoriamente, a lo largo de 8 semanas, una serie de inyecciones intramusculares de oxígeno/ozono en 17 pacientes tratados con antiinflamatorios no esteroideos (72). El mismo grupo de investigación en 2010 comparó las infiltraciones aisladas de $\mathrm{O}_{2} / \mathrm{O}_{3}(3 \mathrm{ml}$ a $10 \mu \mathrm{g} / \mathrm{ml})$ con las infiltraciones más ozonoterapia sistémica rectal en dos grupos de pacientes con artritis reumatoide de la articulación temporomandibular; las mejorías, tanto en el dolor, como en la función y en el estado de la cápsula articular, fueron estadísticamente significativas a favor de la terapia combinada (73). A este respecto, la ozonoterapia sistémica parece disminuir los niveles de interleukina 1 beta (74), directamente relacionados con la actividad de la enfermedad (75), mientras que la ozonoterapia intraarticular disminuiría los niveles de interleukina 8 dentro de la articulación, justificando el menor recuento de granulocitos (76) y la mejoría clínica.

Con respecto a la fibromialgia, esta es una "enfermedad del estrés" en la que subyace una alteración del balance de óxido-reducción celular, consecuencia de un aumento de la producción de radicales libres, una deficiencia de la capacidad antioxidante orgánica o de ambas circunstancias simultáneamente. Los hallazgos bioquímicos soportan esta realidad (77) y la ozonoterapia sistémica ha sido propuesta como tratamiento (78). Hidalgo y colaboradores, en 1996, trataron con 10 sesiones de autohemoterapia a 21 pacientes con fibromialgia refractaria a un plan de tratamiento multidisciplinario, encontrando muy buena tolerabilidad y mejoría en el dolor y la fatiga, así como una disminución significativa en el uso de medicamentos para el dolor (79). Este mismo grupo ha administrado una dosis por vía rectal ( $200 \mathrm{ml}$ de gas a $40 \mu \mathrm{g} / \mathrm{ml}$ ) durante 24 sesiones a 36 pacientes con fibromialgia, recogiendo mejorías significativas en el FIQ (Fibromyalgia Impact Questionnaire), en el estado anímico y en el componente físico del test de calidad de vida SF-12. El tratamiento fue muy bien tolerado, con meteorismo transitorio como efecto adverso más relevante (80).

\section{CONTRAINDICACIONES DE LA OZONOTERAPIA}

Todos los autores coinciden en la seguridad de los tratamientos con ozonoterapia, especialmente ahora que se dispone de máquinas modernas de gran precisión.

Jacobs, en Alemania, publicó que la incidencia de efectos adversos de la ozonoterapia sistémica era de solo el 0,0007\%, llamando la atención las náuseas, el dolor de cabeza y la fatiga (81). En Cuba, con una experiencia de 25 años, teniendo como mínimo una unidad de ozonoterapia por cada provincia del país, solo se han registrado efectos adversos ligeros (82).

La experiencia de los expertos italianos es similar, aunque el Dr. Bocci describe al menos seis muertes por aplicaciones del gas de manera intravenosa directa, práctica absolutamente prohibida en la Unión Europea (9).

Eventualmente, el efecto adverso más serio sería una reacción vagal, generalmente asociada al dolor durante la infiltración, y hay que tener en cuenta que esta ha de ser lenta, especialmente si se va a infiltrar un gran volumen de gas a una concentración elevada (83).

Como contraindicación absoluta está el déficit de glucosa-6-fosfatodehidrogenasa (favismo), ya que este enzima es necesario para abastecer de hidrogeniones al sistema glutatión, encargado de tamponar la oxidación que los lipoperóxidos producirán en el hematíe (84).

Como contraindicaciones relativas para la ozonoterapia sistémica estarían el hipertiroidismo no controlado, la trombocitopenia, la inestabilidad cardiovascular severa y los estados convulsivos. Tampoco es conveniente, por prudencia médica, aplicar la ozonoterapia sistémica a pacientes embarazadas (85).

Las infiltraciones se deberán evitar en cuadros hemorrágicos en pacientes anticoagulados. 
Indudablemente, la ozonoterapia ha de ser practicada por un médico experto, y sería deseable un diagnóstico del estado prooxidante-antioxidante del paciente.

En cuanto a la genotoxicidad del oxígeno/ozono médico, se han realizado muchos estudios que prueban su absoluta seguridad a dosis terapéuticas, salvo por vía inhalatoria, totalmente prohibida por su potencial tóxico (8). Como ejemplo, sirva un estudio llevado a cabo en Cuba, en el que Fernández y su equipo estudiaron la citotoxicidad del ozono, sin ninguna respuesta agresiva, a dosis hasta 10 veces superiores a la máxima dosis terapéutica empleada en autohemoterapia en humanos (86).

\section{CORRESPONDENCIA:}

Francisco Javier Hidalgo-Tallón

e-mail: fjavierclinalgia@gmail.com

\section{BIBLIOGRAFÍA}

1. Schwartz A, Kontorchnikova K, Malesnikov O. Guía para el uso médico del ozono. Fundamentos terapéuticos e indicaciones. AEPROMO; 2011.

2. Pressman S y Warburg OH. The story of ozone. 6th ed. Plasmafire; 2000.

3. Ajamieh HH, Berlanga J, Merino N, Martínez-Sánchez G, Popov I, Menéndez S, Giuliano A, Re L, León OS. Role of protein synthesis in the protection conferred by ozone-oxidative-preconditioning in hepatic ischaemia/reperfusion. Transpl Int 2005;18:1-9.

4. Barber E, Menéndez S, Barber MO, Merino N, Calunga JL. Estudio renal funcional y morfológico en riñones de ratas pretratadas con ozono y sometidas a isquemia caliente. Revista CENIC Ciencias Biológicas 1998;29:178-81.

5. Ajamieh HH, Menéndez S, Martínez-Sánchez G, Candelario-Jalil E, Re L, Giuliano A, León OS. Effects of ozone oxidative preconditioning on nitric oxide generation and cellular redox balance in a rat model of hepatic ischaemia-reperfusion. Liver Int 2004;24:55-62.

6. Ajamieh HH, Menéndez S, Merino N, Martínez G, Re L, León OS. Ischemic and ozone oxidative preconditioning in the protection against hepatic ischemic-reperfusion injury. Ozone Sci \& Eng 2003;25:241-50.

7. Peralta C, Xaus C, Bartrons R, León OS, Gelpi E, Roselló-Catafau J. Effect of ozone treatment on reactive oxygen species and adenosine production during hepatic ischemia-reperfusion. Free Rad Res 2000;33:595-605.

8. Menéndez S, et al. Ozono. Aspectos básicos y aplicaciones clínicas. Centro de Investigaciones del Ozono CENIC 2008; Ciudad de La Habana, Cuba.

9. Bocci V. Oxygen-Ozone Therapy. A critical evaluation. Dordrecht, The Netherlands: Kluwer Academic Publishers; 2002.

10. Verga C. Nuovo approccio terapeutico alle ernie protusioni discali lombari. Riv Neuroradiol 1989;2:1489.

11. Carmona L. Revisión sistemática: Ozonoterapia en enfermedades reumáticas. Reumatol Clin 2006;2:119-23.

12. Iliakis E. Utilizzo dell'ossigeno-ozonoterapia nella pratica ortopedica. Acta Toxic. Ther 1996; vol XVII:249-53.
13. Benvenuti P. Oxygen-Ozone Treatment of the Knee, Shoulder and Hip. A Personal Experience. Rivista Italiana di Ossigeno-Ozonoterapia 2006;5:135-44.

14. Riva-Sanseverino E. Knee-joint Disorders Treated by oxygen-ozone therapy. Europa Medicophysica 1989;25:163-70.

15. Escarpanter-Buliés JC, Valdés-Díaz O, Sánchez-Rauder R, López-Valdés Y, López-García C. Resultados terapéuticos en la osteoartritis de la rodilla con infiltraciones de ozono. Rev Cubana Invest Bioméd 1997;16(2).

16. Manzi R and Raimondi D. The role of oxygen-ozone therapy in patellofemoral chondromalacia. Rivista Italiana di Ossigeno-Ozonoterapia 2002;1:31-5.

17. Gjonovich A, Marchetto R, Montemarà E, Girotto T. Refractory tendinopathies of the knee: Use of oxygen-ozone therapy. Rivista Italiana di ossigeno-Ozonoterapia 2003;2:187-92.

18. Gjonovich A, Girotto T, Montemarà E. Jumper's knee: Oxygen-ozone therapy in refractory forms. Clinical experience. Rivista italiana di Ossigeno-Ozonoterapia 2002;1:183-18.

19. Moretti B, Lanzisera R, Morese A, Moretti L, Patella S, Patella V, Simone C. O2-O3 vs. chondroprotectors in the treatment of osteoarthritis of the knee. Rivista Italiana di Ossigeno-Ozonoterapia 2004;3:65-72.

20. Ikonomidis ST, Iliakis EM, Charalambus Dvakirtzian L. Nonoperative treatment of shoulder impingement syndrome with topical injections of medical oxygen-ozone mixture. A double blind clinical trial. Rivista Italiana di Ossigeno-Ozonoterapia 2002;1:41-4.

21. Trenti GF, Gheza G. Efficacy of oxygen-ozone pain therapy associated with shock waves to treat calcifying tendinitis of the shoulder. Preliminary findings. Rivista Italiana di Ossigeno-Ozonoterapia 2002;1:45-50.

22. Brina L, Villani PC. Treatment of rotator cuff lesions with echo-guided infiltration o fan oxygen-ozone mixture. Rivista Italiana di Ossigeno-Ozonoterapia 2004;3:139-47.

23. Albertini F. Ozone administration in the treatment of herniated cervical disc. Case report. Rivista Italiana di Ossigeno-Ozonoterapia 2002;1:203-6.

24. Villa G. C6-C7 herniated disc treatment with paravertebral oxygen-ozone infiltration. Rivista Italiana di Ossigeno-Ozonoterapia 2002;1:199-201.

25. Moretti B, Lanzisera R, Pesce V, Moretti L, Patella S, Patella V. Simone C. O2-O3 vs. anti-inflammatory drugs in the treatment of neck pain. Rivista Italiana di Ossigeno-Ozonoterapia 2004;3:131-7.

26. Yue Yong Xiao, Jin Lin Tian, Jia Kai Li, Jin Shan Zhang. CT.Guided ozone injection for the treatment of cervical disc herniation. Rivista Italiana di Ossigeno-Ozonoterapia 2006;5:109-15.

27. Muto M, Ambrosanio G, Guarnieri G, Capobianco E, Piccolo G, Annunziata G, Rotondo A. Low back pain and sciatica: Treatment with intradiscal-intraforaminal O2-O3 infection. Our experience. Radiol Med 2008;113:695-706.

28. Cosma F, Simonetti L, De Santis F, Agati R, Ricci R, Leonardi M. Minimally invasive oxigen-ozone therapy for lumbar disk herniation. Am J Neurol 2003;24:996-1000.

29. Bonetti M, Fontana A, Cotticelli B, Dalla-Volta G, Guindani $\mathrm{M}$, Leonardi M. Intraforaminal O2/O3 versus periradicular steroidal infiltrations in lower back pain: Randomized controlled study. Am J Neurol 2005;26:996-1000.

30. Torres LM, Terrero MJ, Vidal M, Aragón F, Martínez J. Discólisis con ozono intradiscal en el tratamiento de la ciática por hernia discal. Rev Soc Esp Dolor 2009;16:147-52.

31. Buric J, Alexandre A, Corò L, Azuelos A. Intradiscal ozone treatment of non-contained disc herniations. 18 months 
follow-up. Rivista Italiana di Ossigeno-Ozonoterapia 2003;2:153-60.

32. Buric J. Ozone chemyonucleolysis vs microdiscectoy. Prospective controlled study with 18 months follow-up. Rivista Italiana di Ossigeno-Ozonoterapia 2005;4:49-54.

33. Qing H, Feng D, Tao L, Hui L, Xiao Fang L, Dong L. Report on 602 cases of percutaneous ozone puncture chemonucleolysis treating lumbar disc protusion. Rivista Italiana di Ossigeno-Ozonoterapia 2005;4:145-8.

34. Castro M, Cánovas L, Martínez J, Pastor A, Segado I, Rocha F, Izquierdo C. Discólisis percutánea con ozono: nuestra experiencia. Rev Soc Esp Dolor 2009;16:405-9.

35. Zambello A, Fara B, Tabaracci G, Bianchi M. Epidural steroid injection vs. paravertebral O2/O3infiltration for symptomatic herniated disc refractory to conventional treatment. A prospective randomized study. Rivista Italiana di Ossigeno-Ozonoterapia 2006;5:123-7.

36. Paoloni M, Di Sante L, Caccio A, Apuzzo D, Marotta S, Razzano M, Franzini M, Santilli V. Intramuscular oxygen-ozone therapy in the treatment of acute back pain with lumbar disc herniation: A multicenter, randomized, double-blind, clinical trial of active and simulated lumbar paravertebral injection. Spine 2009;34:1337-44.

37. Stephen J, Thomas-Meaders BS, Muto M, Murphy KJ. A metaanalysis of the effectiveness and safety of ozone treatments for herniated lumbar discs. Journal of vascular and interventional radiology 2010;21:534-48.

38. Lory MD. Decision making in lumbar disc disease. Clinical Neurosurg 1991;39:36-51.

39. Gangi A, Dietemann JL, Mortazavi R, Pfleger D, Kauff C, Ray C. CT-guided interventional procederes for pain management in the lumbosacral spine. Radiographics 1998;18:621-33.

40. Manchikanti L, Singh V, Cash KA, Pampati V, Datta S. Preliminary results of a randomized, equivalence trial of fluoroscopic caudal epidural injections in managing chronic low back pain: Part 3 - post surgery syndrome. Pain Physician 2008;11:817-31.

41. Andreula CF, Simonetti L, de Santis F, Agati R,Ricci R, Leonardi M. Minimally invasive oxygen-ozone therapy for lumbar disk herniation. Am J Neuroradiol 2003;24:996-1000.

42. Hernández-Guinea BD, Tenopala-Villegas S, Canseco-Aguilar CP, Torres-Huerta JC. Eficacia de la aplicación de ozono epidural y paravertebral a una concentración de $30 \mu \mathrm{gr} / \mathrm{ml}$ para el manejo del dolor crónico en pacientes con síndrome de cirugía fallida de espalda. Rev Soc Esp Dolor 2012;19:310.

43. Riestra-Grijalva YM, Hernández-Santos JR, Tenopala-Villegas S, Canseco-Aguilar CP, Torres-Huerta JC. Eficacia de la aplicación de ozono epidural y paravertebral a una concentración de $50 \mu \mathrm{gr} / \mathrm{ml}$ en pacientes con dolor crónico secundario a síndrome postlaminectomía. Rev Soc Esp Dolor 2012;19:66-71.

44. Yu B, Chen HQ, Lu CH, et al. Effects of different concentrations ozone on synovitis in rat with rheumatoid artritis. Orthopedic Journal of China 2011-12.

45. Moretti B, Lanzisera R, Sisti GL, Moretti L, Patella S, Patella V, Simone C. O2-O3 therapy in tendinopathies and entrapment syndromes. Rivista Italiana di Ossigeno-Ozonoterapia 2005;4:20-9.

46. Ikonomidis ST, Iliakis EM, Eleftheriadou A, Bratanis D, Thomaidis R. Conservative treatment of acute or chronic tendinitis with oxygen-ozone mixture. A double blind clinical trial. Rivista Italiana di Ossigeno-Ozonoterapia 2003;2:67-71.
47. Gaffuri M, Garaffo R, Gheza G. Oxygen-ozone therapy for lateral humeral epicondylitis: Preliminary findings. Rivista Italiana di Ossigeno-Ozonoterapia 2003;2:169-72.

48. Bonetti M. CT-guided oxygen-ozone infiltration into isthmic lysis points in the management of 1st negree spondylolisthesis and spondylolysis. Rivista Italiana di Ossigeno-Ozonoterapia 2003;2:31-8.

49. Alvarado R. Ozonetherapy to resolve disc space infection spondylodiscitis. Rivista Italiana di Ossigeno-Ozonoterapia 2006;5:117-21

50. Bonetti M, Cotticelly B, Richelmi P, Valdenassi L. Rofecoxib and $\mathrm{O} 2 / \mathrm{O} 3$ therapy vs. O2/O3 therapy in the Management of spondyloarthrosis. Rivista Italiana di Ossigeno-Ozonoterapia 2002;1:171-8.

51. Zanardi G, Zorandi A. Oxygen-ozone therapy in painful pathologies of the foot of atletes. Rivista Italiana di Ossigeno-Ozonoterapia 2002;1:51-6.

52. Gheza G, Ipprio L, Bissolotti L. Oxygen-ozone therapy in a diabetic patient with De Quervain's tenosynovitis associated with Joint arthrosis. Rivista Italiana di Ossigeno-Ozonoterapia 2002;1:189-92.

53. Gheza G. Metatarsalgia treated with $\mathrm{O} 2-\mathrm{O} 3$ in a patient with post-surgical cicatricial fibrosis alter resection of Morton's neuroma. Rivista Italiana di Ossigeno-Ozonoterapia 2002;1:109-11.

54. Simonetti V, Liprandi B, Musso C, Simonetti M, Strumia K. Ozone therapy about temporomandibular articulation pathologies and ost-disc-radicular pathologies. Corrispondence to Dr. Vincenzo Simonetti, Via Lamarmora 43, 10128-Torino, Italy, Email: v.simonetti@tiscali.it.

55. Siemsen $\mathrm{CH}$. Ozon-Anwendung bei akuten und chronischen Gelenkerkrankungen, in Ozon-Handbuch. Grundlagen. Prävention. Therapie 1995 (E.G. Beck, and R Viebahn-Hänsler, Eds), Ecomed, Landsberg, p. V-9.2-V-9.2 14.

56. Balkanyi A. The treatment of muscle contracture with ozone. Zurich, Switzerland. http:/www.o3center.org/Abstracts/ index.html.

57. Re L. Clinical evidence of ozone interaction with pain mediators. Saudi Med J 2010;31:1963-7.

58. Fuccio $\mathrm{C}$ et al. A single subcutaneous injection of ozone prevents allodyna and decreases the over-expression of pro-inflammatory caspases in the orbito-frontal cortex of neuropathic mice. Eur J Pharmacol 2009;603:42-9.

59. Mc Cord JM. Free radicals and inflammation: Protection of synovial fluid by superoxide dismutase. Science 1974;185:529-31.

60. Curran SF, Amoruso MA, Goldstein BD, Berg RA. Degradation of soluble collagen by ozone or hydroxil radicals. FEBS Lett 1984;176:155-60.

61. Hawkins CL and Davies MJ. Direct detection and identification of radical generated during the htdroxyl radical-induced degradation of hyaluronic acid and related material. Free Rad Biol Med 1996;21:275-90.

62. Bocci V, Pogni R, Corradeschi F, Busi E, Cervelli C, Bocchi L, Basosi R. Oxygen-ozone in orthopaedics: EPR detection of hydroxyl free radicals in ozone-treated "nucleus pulposus" material. Riv Neuroradiol 2001;14:55-9.

63. Leonardi M, Simonetti L, Barbara C. Effetti dell'ozono sul nucleo pulposo: Reperti anatomo-patologici su un caso operato. Riv Neuroradiol 2001;14:57-9.

64. Iliakis E, Valadakis V, Vynios DH, Tsiganos CP, Agapitos E. Rationalization of the activity of medical ozone on intervertebral disc - A histological and biochemical study. Rivista Di Neuroradiologia 2001;14(Supl. 1):23-30. 
65. Trippel SB. Growth factor actions on articular cartilage. J Rheumatol 1995;43:129-32.

66. Qi WN and Scully SP. Extracellular collagen modulates the regulation of chondrocytes by transforming growth factor-b1. J Orthopaed Res 1997;15:483-90.

67. Gao X, Kim HK, Chung JM, Chung K. Reactive oxygen species (ROS) are involved in enhancement of NMDA-receptor phosphorilation in animal models of pain. Pain 2007;131:262-71.

68. Schwartz ES, Kim HY, Wang J, Lee I, Klann E, Chung JM, Chung K. Persistent pain is dependent on spinal mitocondrial antioxidant levels. J Neurosci 2009;29:159-68.

69. Mattassi R, Bassi P, D’Angelo F, Franchina Asbrascini S. Ozone as therapy in herpes simplex and herpes zoster disease. Medical Application of Ozone. Norwalk: J. LaRaus, Ed.; 1985. International Ozone Association, p. 134-7.

70. Delgado J, Wong R, Menéndez S, Gómez M. Tratamiento con ozono del herpes zóster. Rev. CENIC C Biol 1989;20:160-1.

71. Konrad H. Ozone therapy for postherpetic neuralgia. A retrospective study of 55 cases. Proceedings of the 15th Ozone World Congress, London, UK, 11th-15th September 2001. Medical Therapy Conference (IOA 2001, Ed.), Speedprint MacMedia Ltd, Ealing, London UK, p. 85-8.

72. Menéndez F, Díaz G, Menéndez S. Ozonoterapia en la artritis reumatoidea. Revista CENIC Ciencias Biológicas 1989;20:144-51.

73. Méndez-Pérez I, Cerro-Montesino A, Cámbara-Peña R, Martínez-Godine J, Menéndez-Cepero S. Ozonoterapia sistémica e intra-articular en la artritis de la articulación temporomandibular por artritis reumatoide. Revista CENIC. Ciencias Biológicas 2010;41:169-72.

74. Fahmy Z. Correlation of plasma interleukin 1 levels with disease activity in rheumatoid arthritis with and without ozone. Proceedings in 2nd International Symposium on Ozone Applications. Habana, Cuba 1997.

75. Eastgate JA, Symons JA, Wood NC, Grinlinton FM, di Giovine FS, Duff GW. Correlation of plasma interleukin 1 levels with disease activity in rheumatoid arthritis. Lancet 1988;2:706-9.

76. Fahmy Z. Inmunological effect of ozone $(\mathrm{O} 2 / \mathrm{O} 3)$ in rheumatic diseases. Proceedings of the Eleventh Ozone World Congress, Ozone in Medicine 1993; p M 2-1 to M 2-8.

77. Hidalgo-Tallón FJ. Fibromialgia. Un trastorno de estrés oxidativo. Rev Soc Esp Dolor 2012;2:95-100.

78. Borrelli E, Bocci V. A novel therapeutic option for chronic fatigue syndrome and fibromialgia. Rivista italiana di Ossigeno-Ozonoterapia 2002;1:149-53.

79. Hidalgo J, Calandre E, Rico Villademoros F, Delgado A, Entrena JM, Menéndez S. Interés del ozono sistémico en el tratamiento de la fibromialgia altamente refractaria. En $1^{\mathrm{a}}$ Jornadas Internacionales de Oxígeno-Ozonoterapia Médica. Jerez de la Frontera, 2006.

80. Hidalgo-Tallón J, Vilchez JS, Menéndez-Cepero S, Rodríguez-López CM, Calandre EP. Ozone therapy as addon treatment in fibromialgia management by rectal insufflation: An open label pilot study. Journal of Alternative and Complementary Medicine 2012;18:1-5.

81. Jacobs MT. Untersuchung Über Zwischenfalle Und Typische Komplikationen In Der Ozon-Sauerstoff-Therapie. Ozo Nachrichten 1982;1:5.

82. Menéndez S et al. Ozono. Aspectos básicos y aplicaciones clínicas. Centro de Investigaciones del Ozono CENIC 2008; Ciudad de La Habana, Cuba; p. 27-36.

83. Zambello A, Bianchi M, Bruno F. Sicurezza in ozonoterapia. Rivista Italiana di Ossigeno-Ozonoterapia 2004;3:25-34.

84. Viebahn R. The use of ozone in medicine, 2nd Rev. Germany: Hang Heidelberg, 1994: 1-148.85.

85. Menéndez S et al. Ozono. Aspectos básicos y aplicaciones clínicas. Centro de Investigaciones del Ozono CENIC 2008; Ciudad de La Habana, Cuba.

86. Fernández SI, Quinzan C, Menéndez S, Gómez M, Acosta P. Estudio del efecto del ozono intratesticular sobre la espermatogénesis. Revista CENIC Ciencias Biológicas 2001;32:59-62. 